\title{
ROLE OF X-RAY-INDUCED TRANSCRIPTS IN ADAPTIVE RESPONSES FOLLOWING X-RAYS
}

\section{Proaress Repert-Year 1.}

DOE/ER/61256--1

DE92. 040814

\section{A. BRIEF INTRODUCTION.}

1. DNA Repair Following lenizing Bediation. DNA repair processes which are stimulated by $X$-ray-induced DNA damage are poorly understood. Two such repair processes, PLDR and sublethal damage repair, may be the same manifestations of a series of common enzymatic steps. PLDR, detined as the survival enhancement observed after subculture delay of cells following ionizing radiation, has two distinct phases based upon DNA lesion repair and survival recovery studies (1). The first phase of PLDR occurs very quickly ( $t_{1 / 2}: 2-20$ mins) to increase the survival of $X$-irradiated cells and to mend the vast array of DNA lesions created by ionizing radiatisn. It is associated with a rapid resealing of single-, and later on, double-stranded DNA lesions which are either created initially by X-rays or produced as a result of the repair of various types of base damage (1). The second slower phase of PLDR proceeds much later (1.e., >1-2 hrs) following X.irradiation, during which the remaining double-stranded DNA breaks are completely repaired. This second phase of repair closely corresponds to the restructuring of gross chromosomal damage, and can be partially blocked in some human cells by inhibiting protein synthesis $(1,2)$. This slower phase of PLDR correlated with a rapid decline in X-ray-induced transformation of normal cells.

In theory, the fast component of PLDR may be due to constitutively synthesized enzymes [such as DNA ligases, topoisomerases, or polymerases (3)], which act immediately to repair damaged DNA. In contrast, the slow phase of PLDR in human cells may require the induction of specific genes and gene products involved in the repair of potentially lethal or carcinogenic DKA lesions, including more complex chromosomal damage. PLDR responses which reiy upon inducible genes and proteins may be further enhanced if mammalian cells were "adapted" via prior exposure to lowdoses of ionizing radiation. Thus, $X$-ray-inducible genes and proteins may play a number of roles in adaptive survival responses in human cells, including cell cycle control, the regulation of DNA repair processes, and the suppression of tumor formation through error-correcting, DNA repair proof-reading activities.

2. Are DNA Repair systems Induced BY ONA Damace? A number of experiments with mammalian cells indicate that inducible physiologic responses (i.e,. the production of new genes/proteins) may affect radioresistance, mutagenesis, and carcinogenesis, although much of the evidence is indirect (4). The response of a given cell to DNA damage may depend upon a number of factors, including: (a) the necessary time required to repair the entire genome; (b) the ability to induce or constitutively synthesize the necessary repair pathways; (c) the extent and type of iritial DNA damage created by the cytotoxic agent; and (d) the cell's state of division. Under the influence of repeated low doses of ionizing radiation, cells may respond by demonstrating an "Adaptive Response" to higher doses of that same cytotoxic agent.

Adaptive survival responses are observed when cells are repeatedly treated with low doses of a cytotoxic agent (i.e., primed) and subsequently challenged with higher doses. "Primed" cells survive better than "unprimed" cells following a high dose challenging event. DNA repair enzymes are thought to play key roles in adapting cells to a given DNA demaging agent.

3. X-Ray-Induced Genes and Proteins Synthesized By Mammallan Cells Eollowing lonizing Radiation. Induced gene products (i.e., proteins) specifically synthesized in response to physiological doses of ionizing radiation in radioresistant 


\section{DISCLAIMER}

This report was prepared as an account of work sponsored by an agency of the Linited States Government. Neither the United States Government not any agency thereof, nor any of their employees, makes aliy warranty, express or implied, or assumes any legal liability or tesponsibility for the accuracy, completeness, or usefulness of any information, apparatus, product, or process disclosed, or represents that its use would not infringe privately ow'ned rights. Reference herein to any specific commercial product, process, or service by trade name, trademark. thanufacturer, or otherwise does not necessarily constitute or imply its endorsement, recommendatiot, or favoring by the United States Government or any agency thereof. The view's and opinions of authors expressed herein do not necessarily state or reflect those of the United States Government or any agency thereof. 
human melanoma (U1-Mel) cells, and in a variety of other human normal and cancer. prone cells, were identified using two-dimensional gel electrophoresis (1). U1-Mel cells were chosen since they have one of the highest PLDR (potentially lethal damage repair) capacities for survival recovery known. We identified and partially characterized ten proteins synthesized by U1-Mel cells. The synthesis of eight of these proteins were specifically induced by ionizing radiation and two proteins were repressed. Neither hoat shock, UV-irradiation, nor bifunctional alkylating agent treatments resulted in the induction of these proteins. The expression of one protein, termed XIP269 to indicate an $X$-ray-Induced Protein of approximately $269 \mathrm{KDa}$, correlated very well $(r=0.89)$ with PLDA capacity (1). This protein was found to be down-regulated by exposure to caffeine or cycloheximide, under conditions in which both PLDF and subsequent adaptive survival responses were prevented (1, 5, Unpublished Data). XIP269 was induced by as little as 5 CGy. It is, therefore, a good candidate for playing a role in DNA repair processes which might involve adaptive survival responses, and this protein is currently being isolated in sufficient quantity for reverse genetic cDNA cloning.

\section{B. PRELIMINARY DATA}

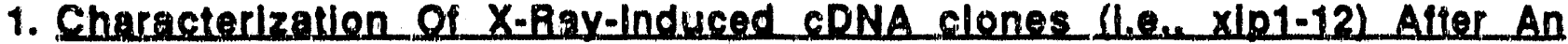
Acute Dose of lonizing Badiation. The data in this section is background preliminary data which characterizes the induction of xip cDNA clones after acute doses of ionizing radiation. It is included in this Progress Report to clarify the induction characteristic of each of the xip clones.

In addition to the characterization of several XIPS, we have isolated twelve X-rayinducible cDNA clones (i.e., xip 1-12) from U1-Mel cells via differential hybridization (6). Some of these genes were induced over 20 -fold above background levels by as little as 5-20 cGy. Using partial DNA sequence data, we have identified tour of these cDNA clones as known human genes: DT Diaphorase, tissue-type Plasminogen Activator $(6,7)$, thymidine kinase, and the proto-oncogene, c-fps/tes. The additional eight cDNA clones (i.e., xip $1,2,4,5,7,8,10$, and 12) represent unidentified gene transcripts, but have only regional homologies to various known genes (6). Table 1 summarizes what we know about these genes at the present time.

We have also investigated the kinetics and specificity of incuction of the xip transcripts using xip cDNA clones as probes on Northern blots. We investigated the temporal expression of xip mRNAs following a single dose of ionizing radiation (i.e., 450 GGy). Confluence-arrested U1-Mel cells were treated with ionizing radiation and at various times RNA was extracted. xip mRNA levels were then monitored by RNA dotblot analyses using individually 32p-labeled xip CDNA insert probes and appropriate loading standard probes (either B-2-microglobulin or the 36B4 homeostasis gene). The levels of induction for xip mRNA transcripts varied from slightly less than 4-fold for xip11 to greater than 226-fold for xip12 (6). Peak levels of xip mRNA transcripts varied in time from 2 hrs to 14 hrs (Table 1).

Without exception, all of the xip mRNA transcripts were transiently induced following ionizing radiation exposure, although their patterns of peak induction varied. With the exception ur an early peak of xip6 (i.e., t-PA), all of the X-ray-induced transcripts were blocked by post-X-ray treatments with actinomycin $D(5 \mu \mathrm{g} / \mathrm{ml})$ and all of the X-rayinduced transcripts demonstrated only one peak over time. As previously described (7), analysis of t.PA mRNA levels revealed two induction peaks, an early peak (appearing between 30 mins and I hour) which was found to be the result of stabilization of its mRNA, and another much larger peak (at 12.14 hours) of t-PA mRNA which was found to be the resutt of new transcription (7).

Times of peak mANA induction for xip transcripts in confluence-arrested U1.Mel cells and in normal human fibroblasts are summarized in Table 1, and ranged irom 1 to 
14 hours. Many of the $X$-ray-inducible transcripts (i.e., xips2, 3, 4, 5, 9, 10, and 11) were expressed within the first 4 hours in both U1-Mel and GM2936B cells following X-ray treatment. Since xip clones were selected from a cDNA library made from U1-Mel cells 4 hours atter $X$-ray exposure and since cDNA probes used in differential hybridization were made from 4 hour time points, it is not surprising that the majority of xip transcripts isolated were expressed at peak levels within the first 4 hours after irradiation.

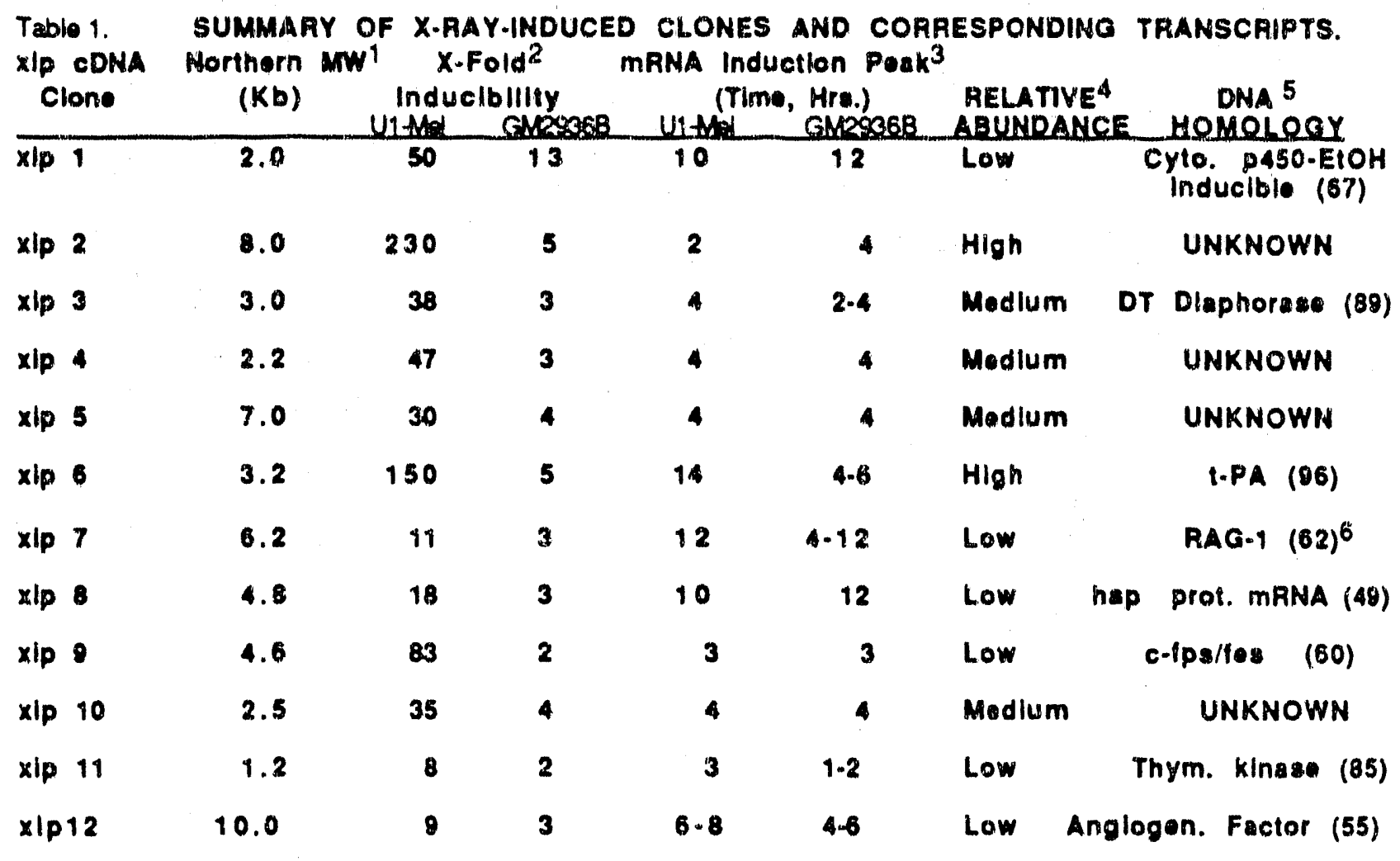

1 RNA Northern blot, dot-blot, and scanning densitometric analyses were pertormed on Zeta-probe strips containing lotal RNA derived trom unirradiatod as compared to iradiated ( 450 cGy) confluence-arrested normal human nontetal fibroblasts (GM2936B) or U1-Mol human molanoma colls as describod in (6).

2 Induction levels (i.e., X-lold) were obtained from RNA dot-blot analyses at times where peak levels of mRNA were noted (6). Helative mRNA ratios wore calculated by dividing the levels of xip mANA transcripts by the mPNA levels of a loading standard, either 36B4 or B-2-microglobulin as described in (6). Ratios oblained from X-irradiated RNA wore compared to those obtained from RNA of unirradiated colls. Ratios ware confirmed by Northern RNA blot analyses when xip mFNA transcripts wore visible.

3 Confluenca-arrested GM2936B or U1-Mel human cells were treated with or wilhout ionizing radiation (450 cGy) and RNA was harvested at various times post-irradiation as described (6). xip mANA lovels were then analyzed via dot-blot. Peak xip mRNA lovels, and times of appearanco, were derived from data presented (6).

4 Represents a subjective measure of the relative abundance of xip mRNAs using t-PA as a relatively abundant transcript toliowing ionizing rediation, and thymidine kinase as a relatively rare MRNA transcript in unirradiated or $X$. irradiated culls.

5. DNA sequence data was obtained from both the $T_{7}$ and $T_{3}$ primers becated on the 3'- and 5'- onds, respectively, of ach xip pBluescript CDNA ckine and analyzed as described in (6). The homology reported represents the percentage of similar DNA sequences to known genes at both ends of the cONA inserts.

6 Recombinant Activating Gene-1.

The expression of xip transcripts was also examined following various doses of ionizing radiation (i.e., dose-response curves for xip expressions were constructed). Confluence-arrested $U 1-\mathrm{Mel}$ cells were exposed to various doses of ionizing radiation and RNA was extracted at periodic intervals thereafter, corresponding to the optimal expression times of each xip transcript. xip transcript expression was subsequently 
monitored. xip transcripts hybridizing to xip cDNA clones, xips1-8, 11, and 12 were induced maximally at a dose of $500 \mathrm{cGy}$. Many of these and other xip transcripts (i.e., xips1, 2, 4, 5, 6, 9, 10, and 12) were induced by as little as $5 \mathrm{cGy}$. Other xip transcripts (i.e., xip3, 7, 8, and 11) were not induced unless U1-Mel cells were exposed to a dose of at least 200 cGy.

We also examined whether or not the xip transcripts were induced by other cytotoxic agents (6)? Confluence-arrested U1-Mel cells were treated with various doses of ionizing radiation and other cytotoxic agents, including UV-irradiation, PMA, and heat shock. U1-Mel cells were exposed to: X-irradiation doses of 200, 500, and 700 cGy; UVirradiation doses of 1, 10, and 30 Joules/M2; PMA doses of 1, 10, and $100 \mathrm{nM}$; and heat shock at $44^{\circ} \mathrm{C}$ for $1 \mathrm{hr}$. The heat shock dose chosen was previously shown to result in the synthesis of several known heat shock proteins $(1,8)$. Doses of UV-irradiation and PMA were chosen since these were previously shown to result in the induction of high levels of t-PA (i.e., xip6) (7). After exposure to these cytotoxic agents, RNA was extracted in a temporal fashion from U1-Mel cells at 0 mins., 10 mins, 30 mins, 2 hrs., 4 hrs., 8 hrs., 12 hrs., 24 hrs., and 48 hrs. After dot-blotting onto nylon membranes, the RNAs were probed with PCR amplified, 32p-labeled xip cDNA inserts. Exposure of U1. Mel cells to ionizing radiation provided the greatest stimulus for the production of xip mRNA transcripts (6). Only t-PA mRNA levels were induced to greater overall levels by UV-irradiation and PMA exposure than by ionizing radiation. MRNA transcripts corresponding to xip4, 7 , and 12 were only induced by ionizing radiation. xip 1, 2, 10 , and 11 wore, for the most part, greatly induced by ionizing radiation, and only slight increases in the production of these mRNAs were observed following UV-irradiation. These X-ray-inducible transcripts were either not affected at all, or only slightly induced by PMA or heat shock treatments during the time frames examined. xip3, 5, and 6 (i.e., tPA) were induced by all of the cytotoxic agents tested, except for heat shock. This is an important finding with respect to adaptive survival responses, since xip5 was one gene whose expression dramatically increased during low dose priming exposures. Since its expression increased in response to several cytotoxic agents, this gene may play a role in the establishment of other forms of adaptive responses to other agents. Both xip3 (i.e., DT diaphorase) and xip11 (i.e., thymidine kinase) were induced by ionizing radiation and UV-irradiation, but not by PMA or heat shock treatments. Only xip8 was induced in response to ionizing radiation and PMA, but not by UV-irradiation. Exposure of U1-Mel cells to heat shock did not result in an increased production of any of the xip mRNA transcripts. Instead, the basal control levels of xip4, 7, 11, and 12 were decreased following heat shock. The remaining xip mRNA transcripts were not affected by heat shock.

Could the induction of these genes and their respective protein products following DNA damage be directly or indirectly involved in DNA repair? Are these gene products also responsible for adaptive survival responses? In the first year of this grant we have begun to investigate these possibilities, as well as to identify additional gene transcripts and products which play a role(s) in mammalian DNA repair processes, which ultimately lead to adaptive survival responses.

2. PRELIMINARY DATA ADDRESSING THE SPECIFIC AIMS. Hypothesis and Specific Aims. The hypothesis that we are testing is that specific gene transcripts are either induced or stabilized following low doses of ionizing radiation. These transcripts resist in the production of new gene products which give rise to an adaptive survival response, 


\section{ultimately leading to Increased radloreslstance as a result of Increased DNA repair processes.}

a. Adaptive Respenses in Different Human Cells. In the first 8 months of this granting period we have made great strides in achieving the goals of Specific Aim \#1. Initially, we asked whether or not an adaptive response could be achieved in all, or a select few, established human cell lines. Two normal human fibroblasts (GM2936B and GM2907A) as well as three human tumor cell lines (U1-Mel melanoma, HEp-2 laryngeal carcinoma, and HTB-152 lung carcinoma) were examined for the ability to establish an adaptive response and for its prevention with cycloheximide treatments (Table 2).:

\begin{tabular}{|c|c|c|c|c|c|}
\hline \multirow{2}{*}{\multicolumn{2}{|c|}{$\begin{array}{c}\text { Table 2. ADAPTIVE } \\
\text { X-RAY CHALLENGE } \\
\text { DOSE } \\
\text { COQA }\end{array}$}} & \multirow{2}{*}{$\begin{array}{l}\text { RESPONSES IN } \\
\text { UNIRRADIATED } \\
\text { CELISS } \\
\text { OHM }\end{array}$} & \multirow{2}{*}{$\begin{array}{l}\text { DIFFERENT } \\
+2 \mathrm{M}\end{array}$} & \multicolumn{2}{|c|}{$\begin{array}{l}\text { HUMAN CELLS } 1 . \\
\text { LOW DOSE-IRAADIATED } \\
\text { CELIS }\end{array}$} \\
\hline & & & & $O M$ & $+C H$ \\
\hline \multicolumn{3}{|c|}{$\begin{array}{cc}\text { Radioresistant U1-Mel Tumor Cells } \\
0.0 & 100 \pm 2.2 \%\end{array}$} & $79 \pm 3.3 \%$ & $91 \pm 3.4 \%$ & $82 \pm 5.2 \%$ \\
\hline \multirow{2}{*}{\multicolumn{2}{|c|}{ HEp-2 $\begin{array}{l}450 \\
\text { Tumor Cells }\end{array}$}} & $24 \pm 1.6 \%$ & $9 \pm 0.9 \%$ & $56 \pm 2.1 \%$ & $12 \pm 1.5 \%$ \\
\hline & & $100 \pm 3.1 \%$ & $85 \pm 4.2 \%$ & $88 \pm 3.8 \%$ & $85 \pm 1.4 \%$ \\
\hline \multirow{2}{*}{\multicolumn{2}{|c|}{$\begin{array}{c}400 \\
\text { HTB-152 Tumor Cells } \\
0.0\end{array}$}} & $17 \pm 0.6 \%$ & $10 \pm 0.2 \%$ & $41 \pm 1.2 \%$ & $12 \pm 0.1 \%$ \\
\hline & & $100 \pm 4.9 \%$ & $79 \pm 3.1 \%$ & $89 \pm 2.3 \%$ & $71 \pm 2.9 \%$ \\
\hline & 400 & $26 \pm 1.3 \%$ & $19+0.4 \%$ & $17+0.3 \%$ & $12+1.0 \%$ \\
\hline
\end{tabular}

GM2936B Normal Ceils

$\begin{array}{crrrr}0.0 & 100 \pm 1.5 \% & 82 \pm 3.4 \% & 93 \pm 5.8 \% & 87 \pm 3.2 \\ 300 & 21 \pm 1.9 \% & 13 \pm 0.6 \% & 26 \pm 0.9 \% & 20 \pm 1.1 \\ \text { GM2907A Normal Cells } & 100+7.2 \% & 93+5.2 \% & 90+4.9 \% & 78 \pm 5.2 \% \\ 0.0 & 18+3.2 \% & 15+2.1 \% & 22+1.1 \% & 16+2.1 \% \\ 350 & 15 \% & \end{array}$

1 Confluence-arrested human cells were exposed to 5.0 cGy each day for 4 days in the presence or absence of $5.0 \mu \mathrm{g} / \mathrm{ml}$ cyctoheximide (CHM). CHM exposures lasted 6 hours after each low dose irradiation, and was removed. Cells were then refed with fresh medium not containing CHM. Low dose-exposed humarn cells, or human cells which were not primed with low dose exposures, were then challenged with a high dose of ionizing radiation. The challenging high doses of ionizing radiation were chosen based upon previous equitoxic measurements (1) Survival levels, as measured via limited dilution colony forming ability, were then perforned. Experiments were pertomed six times. each in duplicalo.

These results indicate that following a series of low dose exposures, U1-Mel cells were able to survive better (i.e., adapt) to a higher challenging dose of ionizing radiation. Confluence-arrested Ui-Mel cells, which were exposed to 4 doses of 5.0 CGy over 4 days, had greater than two times higher surviving fraction following 450 cGy than did a similar population of U1-Mel cells which were not "primed" with prior exposures to low doses of ionizing radiation. Cycloheximide treatments (at a dose of $5.0 \mu \mathrm{g} / \mathrm{ml}$ ), given each day for 6 hours after low dose priming exposures of ionizing radiation, prevented this adaptive response in primed U1-Mel cells. Similar inhibition of the 
adaptive response in U1-Mel cells was observed following continuous cycloheximide exposure $(0.1 \mu \mathrm{g} / \mathrm{ml})$ of low dose irradiated cells. Prolonged exposure of U1-Mel cells to cycloheximide did, however, result in a high degree of cytotoxicity. These preliminary results indicate that U1-Mel cells are able to "adapt" to enhanced survival after repeated challenges to ionizing radiation damage. HEp-2 cells demonstrated a similar adaptive response as that observed with U1-Mel cells. HTB-152 cells, however, did not demonstrate an adaptive response.

In contrast, normal human fibroblast cells were not able to carry out an adaptive response under conditions in which U1-Mel cells did demonstrate such a response. Repeated exposures of $5.0 \mathrm{cGy}$ to normal human fibroblast cells did not result in an overall increase the survival of these cells following an equitoxic high dose challenge. Experiments with other normal human tibroblast cells (i.e., GM2907A and IMR-90) also did not demonstrate a convincing adaptive survival response. In summary, only two out of six cell lines tested have, thus far, demonstrated an adaptive response. in future experiments we will ask "Why do U1-Mel and HEp-2 cells dernonstrate an adaptive response while normal fibroblasts and HTB-452 cells do not?". The answer may be partially explained by the model we have formulated in the next section, which is based upon our initial preliminary data on gene expression changes following adaptive and challenging doses of ionizing radiation. The conditions of growth, the ability of cells to manufacture needed proteins, and the ability of cells to arrest in certain points of the cell cycle are factors which probably lead to the establishment of an adaptive response.

b. Changes in Gene Transcription Following Adaptive and Challenging Deses of lenizing Radiatien. We have identified a number of gene transcripts whose expressions change due to low dose priming (i.e., 5 cGy) exposures and/or after a high dose challenge (i.e., $450 \mathrm{cGy}$ ) (ref. 9). We have also expanded our search for changes in gene expression to include examining protein patterns via two-dimensional gel electrophoresis for changes due to low dose priming and a high dose challenge.

The goals of Specific Aim \#1 were to: test if the X-ray-induced cDNA clones already isolated (Table 1, xip1 to xip 12) were induced by low doses of ionizing radiation, and if they accumulate in human cells upon repeated exposures to small doses of ionizing radiation. Conventional Northern, dot-blot, and nuclear run-on RNA analyses will be utilized to investigate the overall levels of induction and the rates at which these new transcripts are produced following repeated low doses of ianizing radiation. We will also screen low dose-irradiated human cells with probes corresponding to the CDNA which encodes XIP269. We are currently in the process of cloning this cDNA via reverse genetic approaches, beginning with antibodies produced from purified protein trom 2-D gels.

Thus far, we have tested "adapted" or "unadapted" U1-Mel cells for changes in gene expression in four xip cDNA clones (xip1, 3, 5, and 12) and for changes in the expression of variol,s known genes, such as cyclin $A$, cyclin $B$, small nuclear protein, and glutathione-S-transferase. Confluence-arrested U1-Mel cells (grown in twohundred, $150 \mathrm{~mm}^{2}$ tissue culture cishes) were exposed to $5 \mathrm{cGy}$ each day for 4 days to establish a "primed" state. Control cells were treated in an identical manner, but without exposure to ionizing radiation each day. After each priming dose, RNA and protein samples were extracted to be later used in Northern and subtractive hybridization experiments and for two-dimensional gel electrophoretic analysis of protein patteris, respectively. Enough RNA was isolated to identify changes in gene expression usi g both Northern analyses via known (including the xip CDNA clones) CDNA probes and or the isolation of other, more low abundant, gene transcripts which increase in response to various low and/or high dose ionizing radiation stimuli by subtractive hybridization. 
Flow cytometric analyses and $[3 \mathrm{H}]$ thymidine-laboled nuclei measurements were made throughout the experiment to examine the state of cell division of U1-Mel cells during the adaptive and challenging doses of ionizing radiation. Confluence-arrested U1-Mel cells were treated as follows (Figure 1): $X_{1} ;, 5$ cGy on day $1 ; X_{2} ;, 5$ cGy on days 1 and 2; $X_{3}, 5$ cGy on days 1,2 , and $3 ; X_{4}^{\prime}, 5$ cGy on days $1,2,3$, and $4 ; X_{4}^{\prime}, 450,5$ cGy on days $1,2,3,4$, followed by 450 cGy on day $5 ; X_{4}, 450$ + Act $D, 5$ cGy each day on days $1,2,3$, and 4 in the presence of $5 \mu \mathrm{g} / \mathrm{ml}$ actinomycin $D$ for 5 hours post-irradiation, followed by 450 cGy on day 5 in the presence of actinomycin D for 5 hours. X450, 450 cGy to unadapted cells on day $5 ; U_{1}$, unirradiated confluence-arrested $U_{1-M e l}$ cells on day 1 of the experiment; U2, unirradiated confluence-arrested U1-Mel cells on day 5 of the experiment.

1. Changes In xip Transcripts. The expression of xip5 was dramatically altered by low dose priming (Figures 1A-1C). Levels of xip5 mRNA gradually built up after each exposure to the 5 cGy priming doses of ionizing radiation (Figure 1A). Likewise, a mRNA transcript related to xip 12 (a 500 base transcript) gradually built up in response to low dose priming events (Figure $1 \mathrm{C}$ ). The gene transcript corresponding to the original xip12 cDNA clone (i.e., a $10 \mathrm{~Kb}$ ) did not change, except in response to a high dose of ionizing radiation, and the levels of expression of this gene were not different between primed and unprimed cells receiving $450 \mathrm{cGy}$. The overall levels of both xip5 and the xip12-related gene transcripts in primed cells following a high dose challenge were not as high as those observed in unexposed U1-Mel cells following a single high dose (i.e. $450 \mathrm{cGy}$ ) of ionizing radiation (Figures $1 \mathrm{~A}-\mathrm{C}$ ). This may signify a control of gene expression due to low dose exposures. Possibly, low dose primed cells may quickly reach plateau levels of expression of xip5 and xip 12-related genes. Primed cells do not therefore respond in the same way that unirradiated U1-Mel cells do, by inducing massive quantities of xip5 or xip12-related gene products when exposed to high doses of ionizing radiation. Treatment of U1-Mel cells with actinomycin D for 5 hours after each exposure to ionizing radiation prevented the expression of xip5 both during priming, and following a high dose challenge (Figure 1B). These results indicate that new gene transcription, and not mRNA stabilization, is responsible for the dramatic increases in xip5 expression following exposure to ionizing radiation. Similar results were found for the xip12-related transcript (data not shown). 
Figure $1 A-G$

A

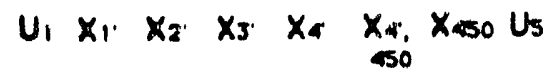

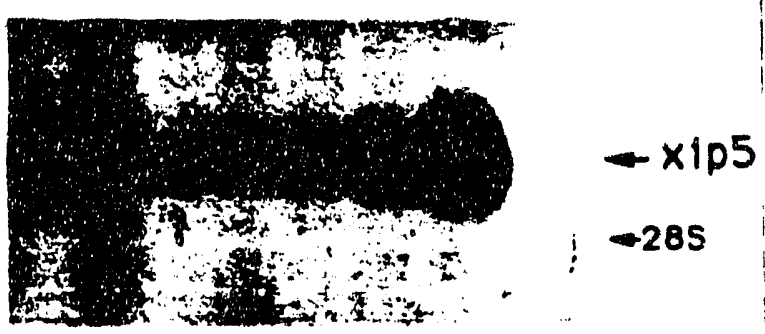

\section{D}

$u_{1} x_{1} x_{2} \quad x_{3} \quad x_{4} x_{4,450} x_{450}$

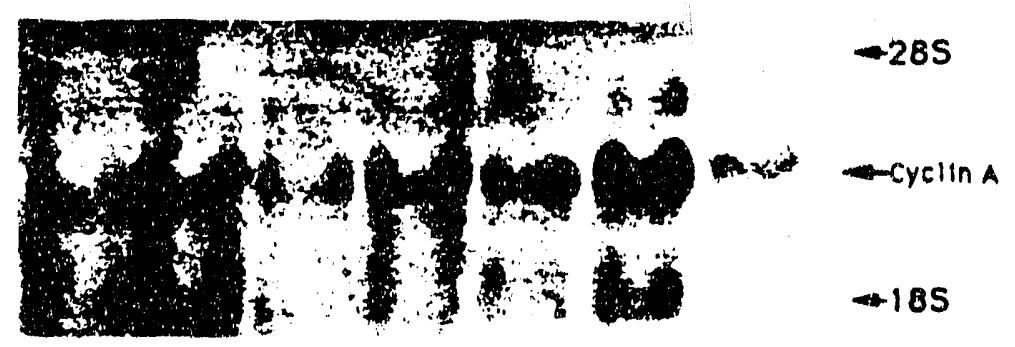

E

$U_{1} x_{1} \quad X_{2} \quad x_{3} \quad x_{4} \quad x_{4,450} x_{450}$ 4
$\rightarrow \cdots+y^{2}$

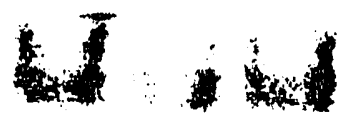

- Cycin 8
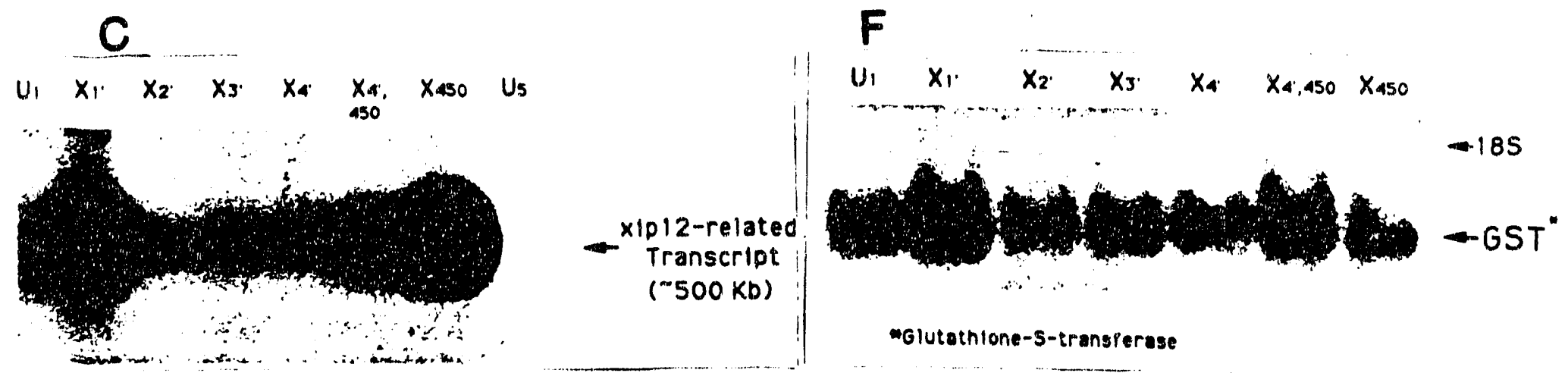

G

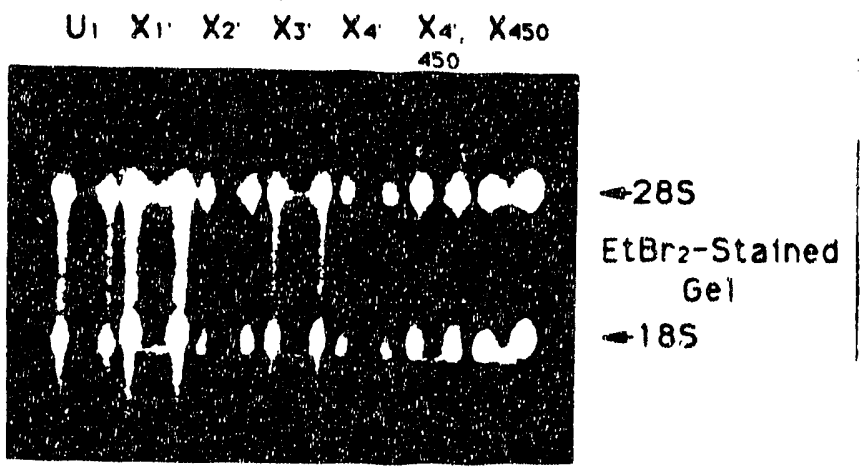


Since the gene for xip5 is being characterized and sequenced we can now ask several questions regarding the gene's function, which were previously outlined in Specific Aim \#3: "What is the function of this gene transcript? Does it control DNA repair and the adaptive response, or is this gene simply the result of a vast number of regulatory changes occurring during low dose exposures to "adapt" the cell for increased repair and survival in response to high dose ionizing radiation exposure. We are in the process of constructing antisense oligos and expression vectors to specifically prevent the low dose induction of this gene. Once we are able to specifically prevent xip5 induction during the adaptive response we will then changes in survival and DNA repair as outlined in Specific Aim \#3.

Similar questions can be asked of the xip12-related gene, only after it's 'ranscript is isolated and cloned. We plan to generate various CDNA probes from the xip12 CDNA clone via restriction enzyme digestion. Once a piece of the xip12 CDNA is found which binds strongly to the 500 bp region in RNA isolated from adapted U1-Mel cells, we will screen for a full length CDNA clone using an adapted, $X$-ray-induced U1-Mel CDNA library, which is being constructed trom the RNA of U1-Mel cells exposed to priming and challenging doses of ionizing radiation as described above. Questions as to the function of the xip12-related CDNA clone may then be asked.

Ii. Changes In Selected Known Gene Transcripts Following lonizing Rediation. We have also used the RNA generated from the adaptive response experiment above to screen for changes in the expressions of various known genes (Figures 1D-1F). Cyclin A, and to a lesser extent, cyclin B were induced in primed cells following a high dose challenge of ionizing radiation (Figures $1 D$ and $1 E$ ). Cyclin genes were examined initially since XIP proteins were known to be cell cycle regulated and since changes in cyclin B were reported following acute doses of ionizing radiation in HeLa cells (10). Interestingly, changes in the expression of either cyclin A or cyclin B were not observed in untreated (i.e., "unadapted") U1-Mel cells, either exposed or not exposed to a challenging high dose of ionizing radiation. Other genes, such as glutathione-S-transierase (Figure $1 F$ ) and the small nuclear protein (not shown) did not change, either following adaptive low dose or high dose challenging exposures. Ethidium bromide stained gels were included to demonstrate small differences in amounts of RNA loaded. For example, lane 2 was loaded with slight more RNA than the other lanes and slight increases in transcript levels in these wells must be compensated for. After densitometric readings of band intensities, cyclin A was found to increase 3fold, while cyclin B levels increased $50 \%$ in primed cells exposed to 450 cGy compared to unadapted cells treated with $450 \mathrm{cGy}$. The data obtained thus far with these gene transcripts can be formulated in a model of how the adaptive response may be achieved in certain cells, and is diagramed in Figure 2 below (ret. 9).

C. Are Changes In Cyelin A and Cyclin B Responsible For The Adaptive Response? A Proposed Model. At present, cyclin A and cyclin B are genes which are known to control the progression of eukaryotic cells through cell division. Yet under the conditions of our experiments, U4-Mel cells did not progress into S-phase as observed by [3H]thymidine-labeled nuclei (Figure 2) and flow cytumetric analyses (not shown). The incuction of cyclin A under these conditions may indicate an involvement of this gene product in stimulating DNA repair, without a full stimulation of cell division. This could be due in part to a lack of all nectssary factors to push U1-Mel cells into cell division (including space limitations) as well as the $X$-ray-inducible expression of certain genes which prevent movement through cell division after DNA damage [e.g., the growth arrest and DNA damage-inducible (gadd) genes $(11,12)]$. Based upon these preliminary data we have developed a model in which an adaptive response may be achieved in mammalian cells (Figure 2).: 
FIGURE 2. Model Explaining the Establishment of An "Adapted" Condition. \begin{tabular}{|l} 
cyclin B mRNA \\
cyclin A mRNA \\
XIP5 MRNA \\
XIP12.RELATED mRNA
\end{tabular}

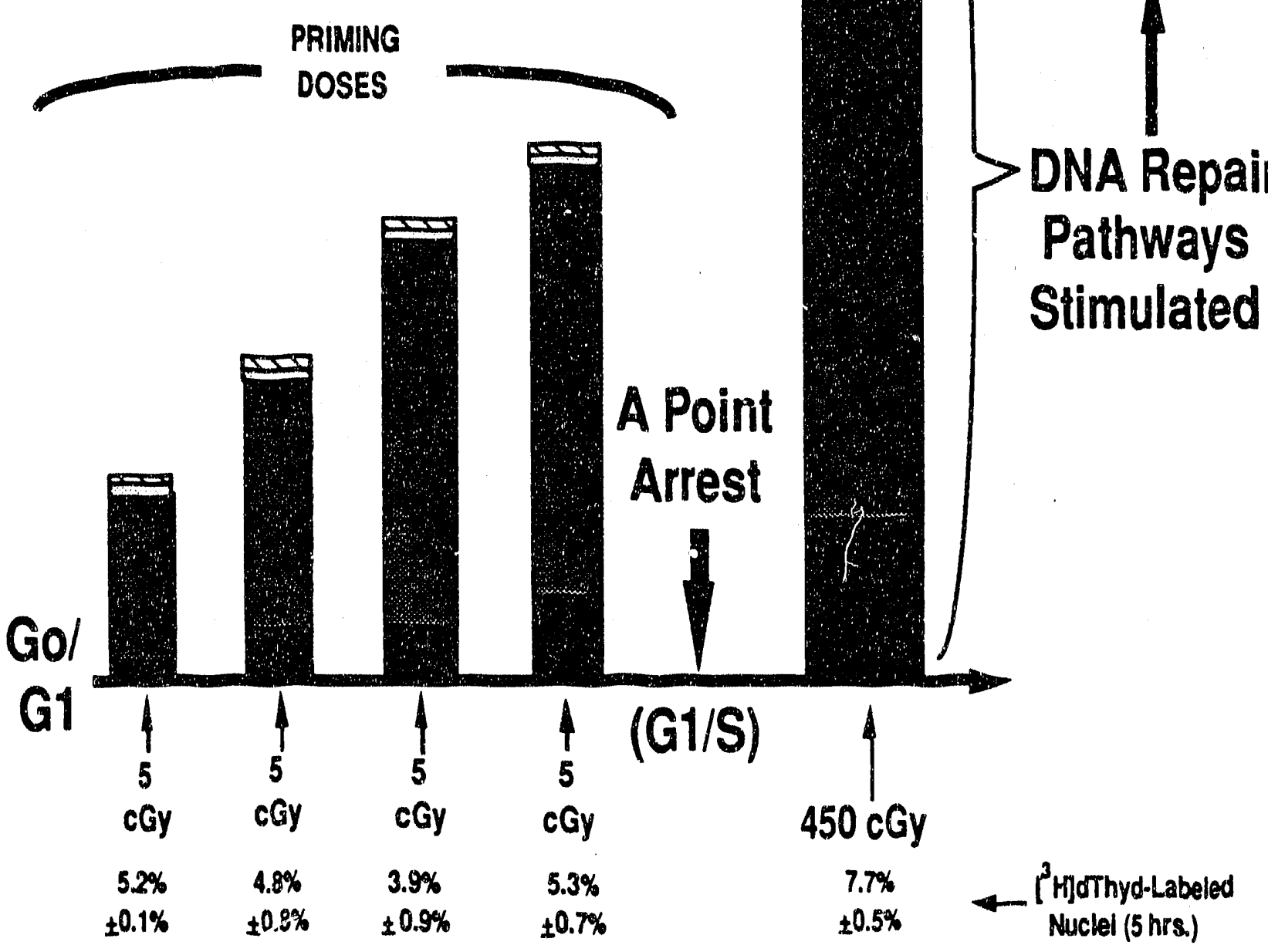

Initially, cells are arrested in $G_{0}$ or at some point in $G_{1}$. Upon repeated exposures to low doses of ionizing radiation (i.e. 5 cGy per day for 4 days) cells progress to, and arrest at, an "A" (for "Adaptive") arrest point at or near the $G_{1} / S$ border of the cell cycle, thus explaining the reacily inducible levels of cyclin $A$ after high dose ionizing radiation exposure; cyclin $A$ levels begin to appear at the $G_{0} / G_{1}$ border (10). It is possible that gene transcripts which build up slowly in response to priming doses (i.e., xip5 and xip12-related mRNAs) produce proteins that regulate or control those transcripts which appear only in response to a high dose challenge (e.g., cyclin A). At the "A" point, cells are "poised" to stimulate various DNA repair systemis which are not inducible in the initial $G_{0} / G_{y}$-arrested state, but are eventually required to establish an "Adaptive Response". We will attempt to test this model using the techniques and experimental plan outlined in Specific Aim \#3. 
D. Future Investigations.

1. Centinued Studies: Year 2.

In the second year of our studies we will continue screening for changes in known genes and xip transcripts using the RNA isolated in Specific Aim \#1. Once a gene transcript has been identified which is affected by low and/or high dose exposures of ionizing radiation we will study these genes in terms of the goals identified in Specific Aim \#3.

In the later part of Year 1 and the early part of Year 2, we will begin to construct CDNA libraries using "primed or adapted" U1-Mel cells either exposed to, or not exposed to, a high dose challenge of 450 cGy ionizing radiation. CDNA libraries from unirradiated U1-Mel cells and from singe-dose-exposed (450 CGy) U1-Mel cells will also be constructed for use in subtractive hybridization screening. We will construct subtracted libraries from primed as compared to unprimed confluence-arrested U1-Mel cells and from primed U1-Mel cells exposed to, or not exposed to, a 450 cGy challenging dose of ionizing radiation.

The experiments described in Specific Aim \#2 will not, however, be completed in the course of this grant. It will take 3 to 5 years to isolate, identify, characterize and determine the function of low abundance gene transcripts which are affected by low dose priming and/or high dose challenging exposures of ionizing radiation. The goals of Specific Aim \#2 were: to use subiractive and/or differential CDNA cloning hybridization techniques to select for, isolate, sequence, and characterize new mRNA transcripts induced following repeated low doses of ionizing radiation as compared to unirradiated or high dose-irradiated human melanoma cells. DNA sequences of the isolated low dose-induced cDNA clones will be obtained, compared to previously sequenced genes via GenBank analyses (including the xip genes 1-12 previously discussed), and their temporal induction levels will be characterized.

The nature of these goals are long-term, and will required additional funding and several "ears to complete. The experiments are essential to better understand the genes which control the adaptive response and DNA repair processes following ionizing radiation. We will, of course, begin these experiments but the results must not be expected for several years. Isolation and characterization of genes controlling the adaptive response will be required to better understand radioresistance, and how it develops in certain human and other mammalian cells.

2. Investigation of The Function(s) of Gene Transcripts Which Vary During The Establishment Of Adapted Conditions. Now that two classes of geres have been found which are alter during low dose exposure, we may now begin to investigate what their functions are during the adaptive response in U1-Mel cells (Figure 1). Class I genes (i.e., xip5 and the xip12-related transcripts) are those that build up slowly in response to low priming doses of ionizing radiation. Class II genes (i.e., cyclin $A$ and B) are those which appear not to be afiected by low dose adapting doses of ionizing radiation, but then appear in response to a high dose challenge. Since Class II genes do not appear after single acute doses of ionizing radiation, these genes may be directly involved in the subsequent radioresistance which accompanies the adaptive response. As outlined previcusly, we will investigate the functions of these genes using the techniques and experimental plan outlined in Specific Aim \#3.

The goals of Specific Aim \#3 were: once low-dose induced cDNA clones have been isolated, we will investigate the function of these cDNA clones via antisense cDNA constructs. The effects of specific gene down-regulation on cell survival recovery (i.e., PLDR), adaptive responses, and physical DNA break repair (using alkaline and neutral filter elution techniques) will be examined. Antisense cDNA constructs corresponding to 
any of the twelve xip clones which demonstrate low dose induction in Specific Aim "1 will be constructed and utilized to determino their involyement in repair and/or radioresistant adaptive responses. Conventional plasmid transfection or a new HSV-1 viral vector capable of holding up to $40 \mathrm{Kbp}$ of inserited CDNA will be utilized for these experiments. (13). Northern blot and nuclear run-on RNA analyses will be utilized to ensure that specific gene down-regulation (corresponding to the chesen xip gene) has occurred. Once candidate CDNA clones thought to be involves in DNA repair have been isolated, complementation studies (via CDNA clone transfection into various cancer-prone celis) will be attempted to investigate the possibility of correcting (probably partial correction will result) individual DNA repair defects in cancer-prone cells. We will initially focus on cloning and functionally characterizing the gene for XIP269 (for which we already have a polyclonal antibody), since it has previously been shown to be involvod in DNA repair processes following ionizing radiation $(1,5)$.

Using CDNA clones for cyclin A and cyclin B as well as for xip5 and the xip12. related CDNA, we hone to begin the experiments outlined in Specific Aim \#3. The CDNA clones generated in specitic Aim \#2 will also be investigated using this experimental plan, when these CDNA clones have been isolated and characterized.

\section{E. Previous Time Frame of investigations.}

As a reminder, I have included my origirial time frame of experimentation in the initial grant proposal. We have made great piogress in the first year of this proposal, yet we have a long way to go in understanding the regulation and function(s) of genes involved in establishing an "Adaptive Response" in certain human colls. Wo will follow the previously described experimental plan to carty out the goals outlined in the figure Dolow (Figure 3).

The following diagram outlines the general research plen of attack described in this proposal for investigating the involvement of mANA transcripts induced by low doses of ionizing radiation in adaptive survival responses.:

Figure \#3.

\section{TIME FRAME OF EXPERIMENTATION}

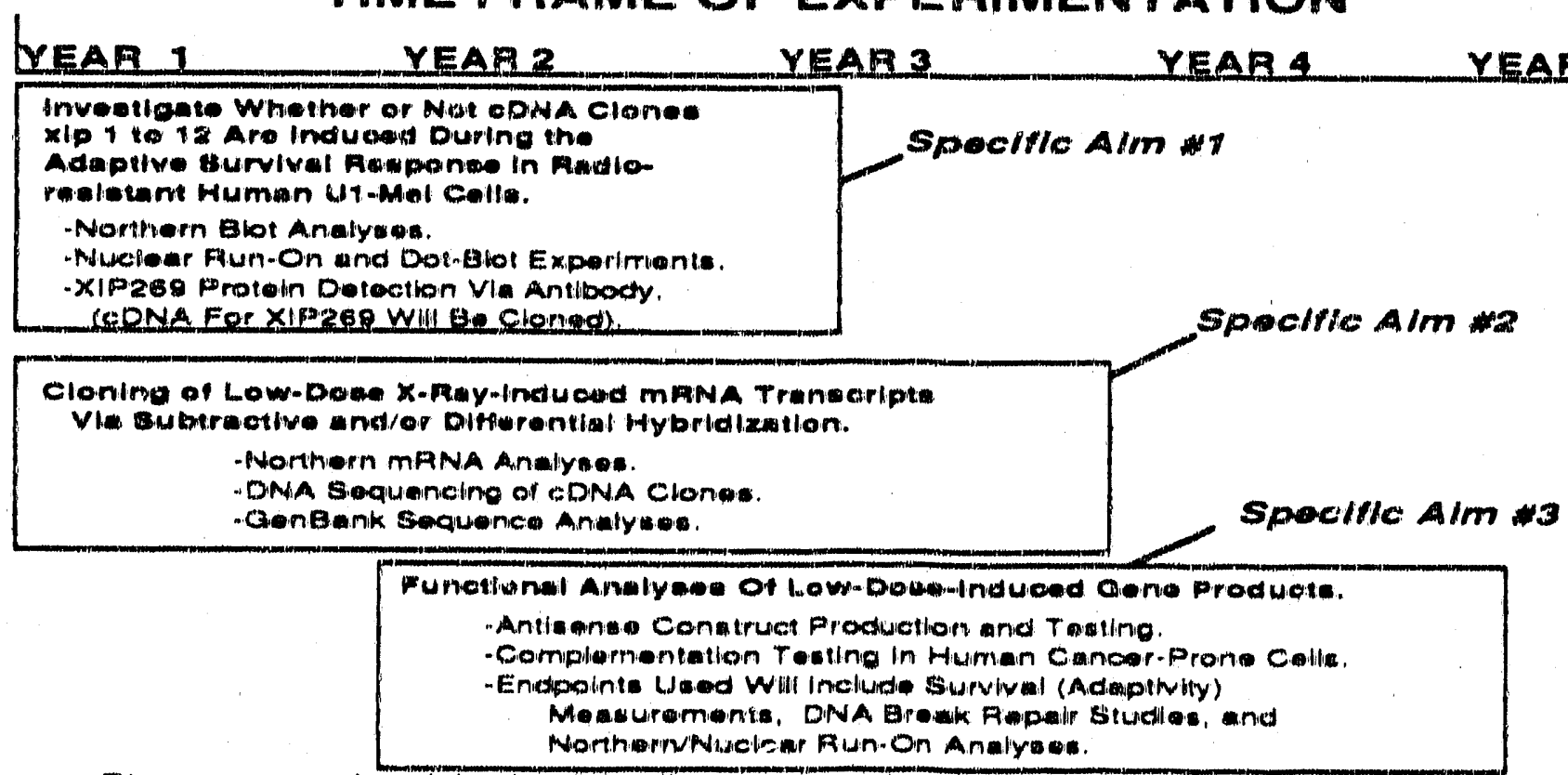

Please note that Thad originally requested a 5 year granting period. The long-term goals of this project will require additional funding, yet at a lower overall annual cost, after the thire year. 
Summary. The overall goal of this proposal is to clone low dose X-ray-induced genes via molecular biology cloning techniques. I have been successful at identiying several genes whose expressions are alter during the establishment of, and actual processes occurring in, the adaptive response of U1-Mel cells. I will, in the next year or so, investigate the kinetics of expression of these genes, and whether or not other environmental stresses affect the expressions of these genes via Northern blot analyses. Finally, I will begin to investigate the function(s) of these genes within the cell, either under normal conditions of growth or following X-irradiation. Corsplementation stuoies, antisense RNA analyses, and DNA repair endpoints (i.e., survival recovery or alkaline/neutral fitter elution studies) will be combined to examine the possibility of correcting the repair deficiencies and enhancing adaptive survival recovery responsus in certain human cancer-prone cells. Since many of the xips identified by 2-D gel electrophoresis were cell cycle regulated (1), the factors turning them on and off within a nurmal cell cycle may be clearly related to the events occurring in human cells following a genetic insult. This theory has been strengthened by our recert findings that cyclin $A$, and to a lesser extent cyclin B, change in response to low dose priming and high dose challenging exposures of ionizing radiation.

F. Human sublec's. None.

G. Yertebrate Animals. Fone in this proposal.

H. Consultants/Collaborators. None.

1. Coneortium/Centractual Arranoements. None.

d. Llterature Clited.

1 Boothman DA, Bouvard I, and Hughes EN. Cancer Res. 49: 2871-2878, 1989.

2. Ronai ZA, Lambert ME, Weinstein IB. Europ. Environ. Occup. Cancer. Cell Biol. Toxicol. 6: 105-126, 1990.

3. Boothman DA, Trask DK, and Pardee AB. Cancer Res. 42: 605-612, 1989.

4. Stein B, Rahmsdorf HJ, Schonthal A, Busher M, Ponta H, and Herrlich P. In: Mechanisms and Consequences of DNA Damage Processing, New Series, (E. Friedberg and P. Hanawalt, Eds.) Alan R. Liss, Inc. New York, NY, 1988.

5. Hughes EN, and Boothman DA. Radiat. Res. 125: 313-317, 1991.

6. Boothman DA, Mcyers M, Fukunaga N, and Lee SW. Isolation and characterization of X-rayinduced transcripts from radioresistant human melanoma cells. Mol. Cell. Biol. (In Preparation), 1991

7. Boothman DA, Wang W, and Lee S. Cancer Res. 51:5587-5595, 1991.

8. Lucke-Hule, C., and Herrlich, P. Int. J. Cancer 39: 94-98, 1987.

9. Meyers M, Schea R, Seabury H, Petrowski A, McLaughlin WP, Lee I, and Boothman DA. Role of $\mathrm{X}$-ray-induced proteins and gene transcripts in adaptive survival responses in radioresistant human melanoma cells. In: "Low Dose Irradiation and Biological Defense Mechanisms", (Takashi Aoyama, Ed.) Elsevier Science Publishers 1992; (In Press).

10. Muschel RJ, Zhang HB, lliakis G, and McKernia WG. Cancer Res. 51:5113-5117, 1991.

11. Fornace AJ, Jr., Zmudzka B, Hollander MC, and Wilson SH. Mol. Cell. Biol. 2: 851-853, 1989.

12. Fornace AJ, Jr., Alamo I, Jr., and Hollander MC. Proc. Nad. Acad. Sci., USA 85: 8800$8804,1988$.

13. Boothman DA, Geller AI, and Pardee AB. FEBS Letters 258: 159.162, 1989. 


\section{APPENDIX ITEMS.}

\section{REPRINTS AND PREPRINTS.}

A. Included below are reprints and preprints of manuscripts and papers resulting from the first year of the Department of Energy grant entitled "Role of X-Ray-Induced Transcripts In Adaptive Survival Responses Following lonizing Radiation".:

1. Fukunaga $N$, Schea $\mathrm{PA}$, Burrows HL, Meyers $M$, and Boothman DA. Enhanced expression of tissue-type plasminogen activator in humal: cancer-prone as compared to normal human fibroblast cells. Int. J. Radiat. Oncol. Biol. Phys., 1992; (In Press).

2. McLaughlin WP, Schea R, McKeever PE, and Boothman DA. Radiobiological effects and changes in gene expression in the central nervous system in response to ionizing radiation. In: "Molecular Genetics of Nervous System Tumors". (Levine, A.J. and Schmidek, H.H. Eds.) Wiley-Liss, Inc. 1992; (In Press).

3. Meyers $M$, Schea $R$, Seabury $H$, Petrowski A, McLaughlin WP, Lee I, and Boothman DA. Role of $X$-ray-induced proteins and gene transcripts in adaptive survival responses in radioresistant human melanoma cells. In: "Low Dose Irradiation and Biological Defense Mechanisms", (Takashi Aoyama, Ed.) Elsevier Science Publishers 1992; (In Press).

B. The following preprints contain information pertinent to the enclosed Progress Report, but are not the result of DOE funding. These manuscripts are available upon request, but have not been included with this DOE Progress Report.:

1. Boothman, D.A., Wang, M., and Lee, S.W. Induction of tissue-type plasminogen activator by ionizing radiation in human malignant melanoma cells. Cancer Res.1991; 51: 5587-5595.

2. Boothman DA, and Lee SW. Regulation of gene expression in mammalian cells following ionizing radiation. Yokohama Medical Bulletin, 1992; 42:137-149.

3. Boothman DA, Meyers $M_{1}$ Fukunaga $N$, and Lee SW. Isclation of complementary DNA species induced by ionizing radiation in human melanoma cells. Molec. \& Cell. Biol., 1992; (In Preparation).

Ii. Original Northern Figures I A-G. 

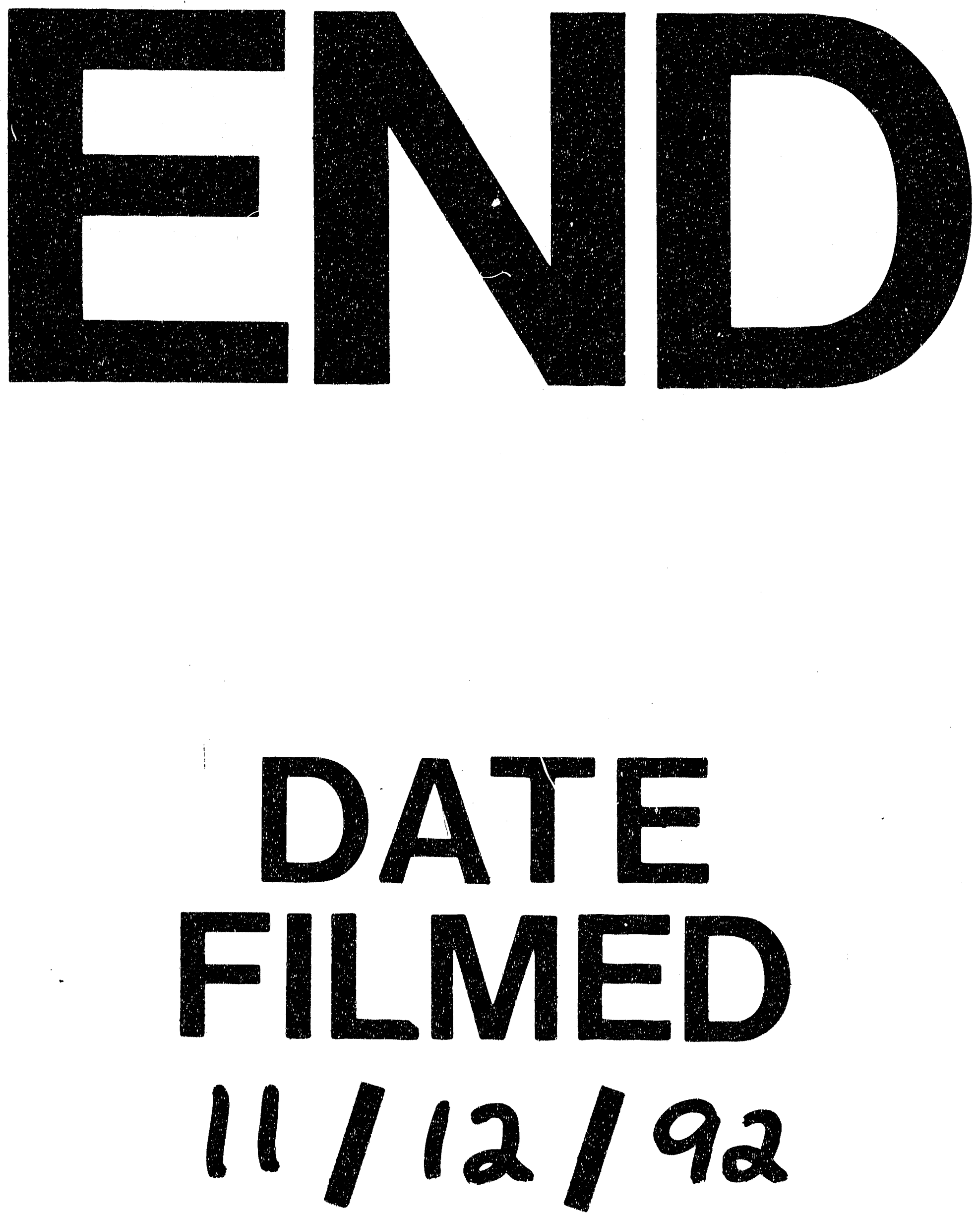

$\frac{1}{\prime \prime}$ 


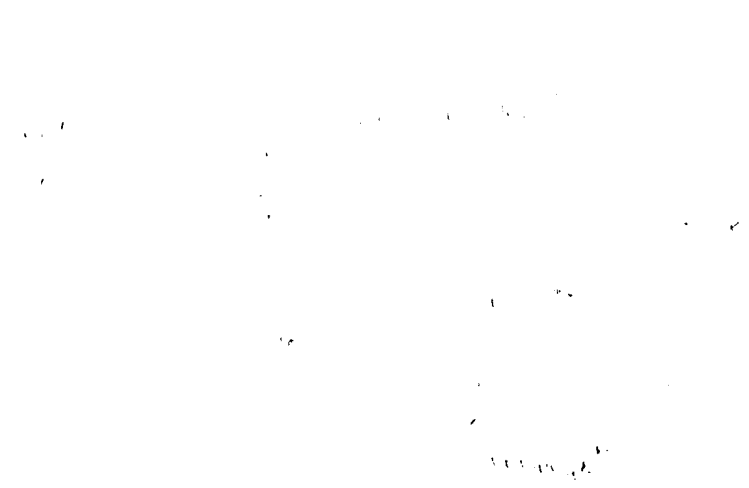

